\title{
ÉTUDE DE L'HABITAT DE LA TRUITE FARIO (SALMO TRUTTA FARIO). RÔLE DES CACHES ARTIFICIELLES ET NATURELLES DANS LES RIVIĖRES AMÉNAGÉES.
}

\author{
P. LIM*, G. SEGURA*, A. BELAUD*, Catherine SABATON**
}

* ENSA Toulouse, 145 avenue de Muret, 31076 TOULOUSE CEDEX

** EDF-DER, 6 quai Watier, 78401 CHATOU CEDEX

\section{RÉSUMÉ}

L'objectif est de quantifier le rôle des abris et caches des rivières aménagées sur le peuplement des truites fario.

L'étude s'est déroulée sur deux tronçons de cours d'eau pyrénéens : la PIQUE (altitude $920 \mathrm{~m}$ NGF) et le GER (altitude 390 m NGF), situés dans le département de la Haute-Garonne (31).

Pour les caches naturelles, nous avons expérimenté sur trois ensembles qui ont pu être transitoirement condamnés par des grillages : enrochement des berges, caves creusées sous les berges, et branchages. Ceci a pu être réalisé sur le GER.

Initialement, le secteur (avec tous ces types de caches) possède une densité moyenne de $2469 \mathrm{ind} / \mathrm{ha}$ pour une biomasse de $278 \mathrm{~kg} / \mathrm{ha}$. Quand l'accès aux caches est condamné, la densité se stabilise à 1000 ind/ha pour une biomasse d'environ $125 \mathrm{~kg} / \mathrm{ha}$, soit une diminution de l'ordre de $55 \%$ en densité et en biomasse. Suivant les types de caches, la recolonisation, après 21 jours de réouverture des caches, est estimée entre 28 et $72 \%$ pour la biomasse et entre 42 et $62 \%$ pour la densité.

Pour les caches artificielles, six types de matériaux de construction (brique, tuile...) ont été expérimentés sur un tronçon de la PIQUE (dénudé de toute cache naturelle). Suivant les types de caches et leur disposition, la densité des truites varie de 3 à $23 \mathrm{ind} / \mathrm{m}^{2}$ de cache, et de 0,09 à 0,2 ind $/ \mathrm{m}^{2}$ hors cache.

Parmi les matériaux utilisés, on observe une nette préférence pour les briques et, selon les dispositions testées, les meilleurs résultats sont obtenus avec les caches placées soit près. des rives, soit dans la totalité du lit.

L'expérience a montré le rôle prépondérant des caches naturelles et artificielles sur le peuplement des truites dans un cours d'eau aménagé.

Mots-clés : truite fario, biomasse, cache, habitat, Pyrénées.

\section{STUDY OF THE HABITAT OF BROWN TROUT (SALMO TRUTTA FARIO). ROLE OF ARTIFICIAL AND NATURAL COVERS ON TROUT POPULATIONS.}

\begin{abstract}
The aim of this study was to quantify the role of various cover types and examine their selection by brown trout.

The study was developed on two river course sections in the Pyrénées : the river PIQUE (altitude $920 \mathrm{~m} \mathrm{NGF}$ ) and the river GER (altitude $390 \mathrm{~m} \mathrm{NGF),} \mathrm{situated} \mathrm{in} \mathrm{the} \mathrm{department} \mathrm{of}$ the Haute-Garonne (31).

For the natural cover, we have experimented on three sets which have been transitionnally blocked by wire netting; rocky river bank, undercut bank and woody debris. This has been performed on the river GER.
\end{abstract}


Initially, the sector, for all these types of covers, had an average density of $2469 \mathrm{ind} / \mathrm{ha}$ for a biomass of $278 \mathrm{~kg} / \mathrm{ha}$. When the access to the covers is protected, the density stabilises to $1000 \mathrm{ind} / \mathrm{ha}$, being a decrease in the order of $55 \%$ in density and biomass. According to the type of cover, the recolonisation, after 21 days of reopening of the covers, is estimated between 28 and $72 \%$ for the biomass and between 42 and $62 \%$ for the density.

For the artificial covers, six types of building materials (brick, tile ...) have been experimented on the river PIQUE section (all natural covers having been removed). By type of covers and their location, the trout density varied from 3 to $23 \mathrm{ind} / \mathrm{m}^{2}$ of cover, and from 0.09 to $0.2 \mathrm{ind} / \mathrm{m}^{2}$ outside cover.

Among the material used, we observed a clear preference for brick and, depending on the dispositions tested, the best results have been obtained with the covers placed near the banks, or being in the totality of the bed.

The experiment has shown the major role the natural and artificial covers have on trout populations.

Key-words : brown trout, biomass, cover, habitat, Pyrénées.

\section{INTRODUCTION}

Les salmonidés vivent généralement dans les lacs et rivières situés en tête de bassin versant. Dans les rivières, la qualité de l'eau est la plupart du temps excellente et l'abondance des poissons dépend largement du débit, de ses variations, de la physico-chimie de l'eau et surtout de la structure physique du lit (BOUSSU, 1954 ; ALLEN, 1969 ; BAIN et al., 1985 ; MILNER et al., 1985 ; BOWLBY et ROFF, 1986). Ces rivières se caractérisent par une succession de pools, de radiers et de rapides avec des diversités de substrats, de profondeurs, de vitesses de courant, constituant ainsi une mosaïque d'habitats.

La truite fario, à un stade de vie donné, aura une préférence pour un type d'habitat qui correspond aux conditions les plus favorables à son développement (DEVORE et WHITE, 1978 ; BINNS et EISERMAN, 1979 ; WILZBACH, 1985 ; CUNJAK et POWER, 1986 ; NIELSEN, 1986 ; RALEIGH et al., 1986 ; GRANT et NOAKES, 1987 ; HEGGENES, 1988 ; HEGGENES et TRAAEN, 1988 ; MCMAHON et HARTMAN, 1989 ; NÄSLUND, 1989).

Cette préférence est appelée classiquement "préférendum d'habitat" qui n'est autre qu'un abri ou une cache (BOVEE, 1982 ; WESCHE et al., 1987 ; BELAUD et al., 1989 ; SOUCHON et al., 1989 ; GRANT et KRAMER, 1990). Les "caches" peuvent être définies comme toute protection pour les poissons contre les forts courants et la prédation. Elles peuvent être de natures diverses : arbres rivulaires, sous-cave, végétation et objets submergés, objets flottants et zones turbulentes (GINGER, 1973). D'autres auteurs comme WILSON (1975), ITO (1978) cités par BACHMAN (1984) définissent la notion de territoire chez les truites en fonction des besoins pour la reproduction, (les refuges, l'alimentation, le rôle des caches plus ou moins important suivant leur emplacement par rapport à l'espace vital).

Notre étude se limitera à préciser le rôle du facteur "cache" sur le peuplement en truites d'un tronçon de rivière. Nous nous intéressons aux caches dites naturelles et aux caches dites artificielles. L'objectif est donc de quantifier le rôle de ces deux types de caches dans les rivières aménagées, sur le peuplement de truite fario. Sur le GER, les aménagements existants sont des enrochements qui protègent la berge (en rive droite) sans modification profonde du fonctionnement physique du cours d'eau. Pour la PIQUE, il existe une succession de seuils, dans un tronçon à débit réservé, créant des chutes d'eau d'environ $5 \mathrm{~m}$ de haut entre chaque bief.

\section{MATÉRIEL ET MÉTHODES}

L'étude s'est déroulée en 1990 et 1991 sur 2 cours d'eau pyrénéens, la PIQUE, rivière à Salmonidés stricte, et le GER à Salmonidés dominants accompagnés de vairons et de chabots, situés dans le département de la Haute-Garonne.

Les caractéristiques succinctes de ces 2 rivières sont données sur la figure 1.

La PIQUE et le GER coulent sur un socle cristallin, contribuant à la faible minéralisation des eaux, la valeur moyenne de la conductivité aux secteurs étudiés est de l'ordre de $80 \mu \mathrm{S} / \mathrm{cm}$ pour la PIQUE et de $105 \mu \mathrm{S} / \mathrm{cm}$ pour le GER. 
Le régime des eaux est de type pluvio-nival avec 2 périodes d'étiage très marquées (été-automne) et une période de hautes eaux lors de la fonte des neiges (printemps).

La PIQUE au secteur étudié est soumise à un débit régulé de l'ordre de $250 \mathrm{l} / \mathrm{s}$ et le GER présente un module de $2,7 \mathrm{~m}^{3} / \mathrm{s}$.
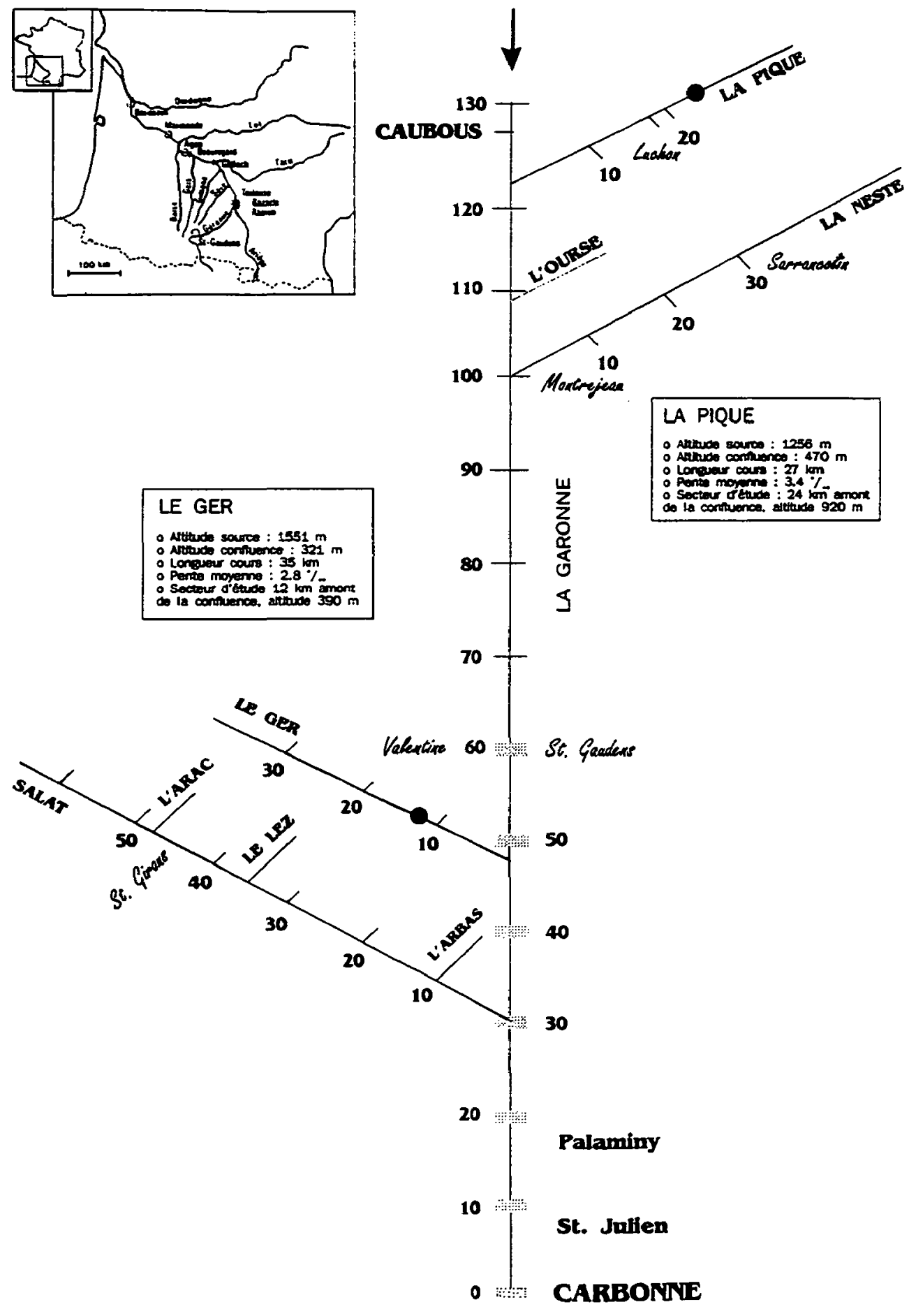

Figure 1 : Situation géographique des cours d'eau étudiés par rapport à l'axe Garonne.

Figure 1 : Map of the Garonne water-system showing locality of studied streams. 


\section{CACHES NATURELLES (fig. 2)}

L'expérience s'est déroulée sur la rivière GER (Haute-Garonne) entre la fin octobre et la fin décembre 1990. Le secteur considéré s'étend sur environ 120 mètres et présente trois groupes de caches principaux :

- ENROCHEMENT : malgré son caractère artificiel, cette entité peut être classée parmi les caches naturelles car elle se rapproche des gros blocs de rochers rencontrés à l'état naturel sur les berges et se situant $2 \mathrm{~km}$ environ en amont du secteur étudié. Située sur la rive gauche, d'une longueur de $37 \mathrm{~m}$ sur $2 \mathrm{~m}$ de large, elle est constituée de gros blocs de protection des berges.

- SOUS-CAVE ou SOUS-BERGE : cette appellation regroupe un abri creusé par le courant sous la berge et surmonté par une souche d'arbre ou par la rive elle-même. Situé sur la rive droite, il mesure 7,5 $\mathrm{m}$ de longueur pour une profondeur de $1,50 \mathrm{~m}$.

- BRANCHAGES : ce terme désigne un entrelacs de branches et de petits troncs d'arbres accumulés le long de la rive. Cette cache, située sur la rive gauche, mesure 10,2 m sur $11 \mathrm{~m}$.

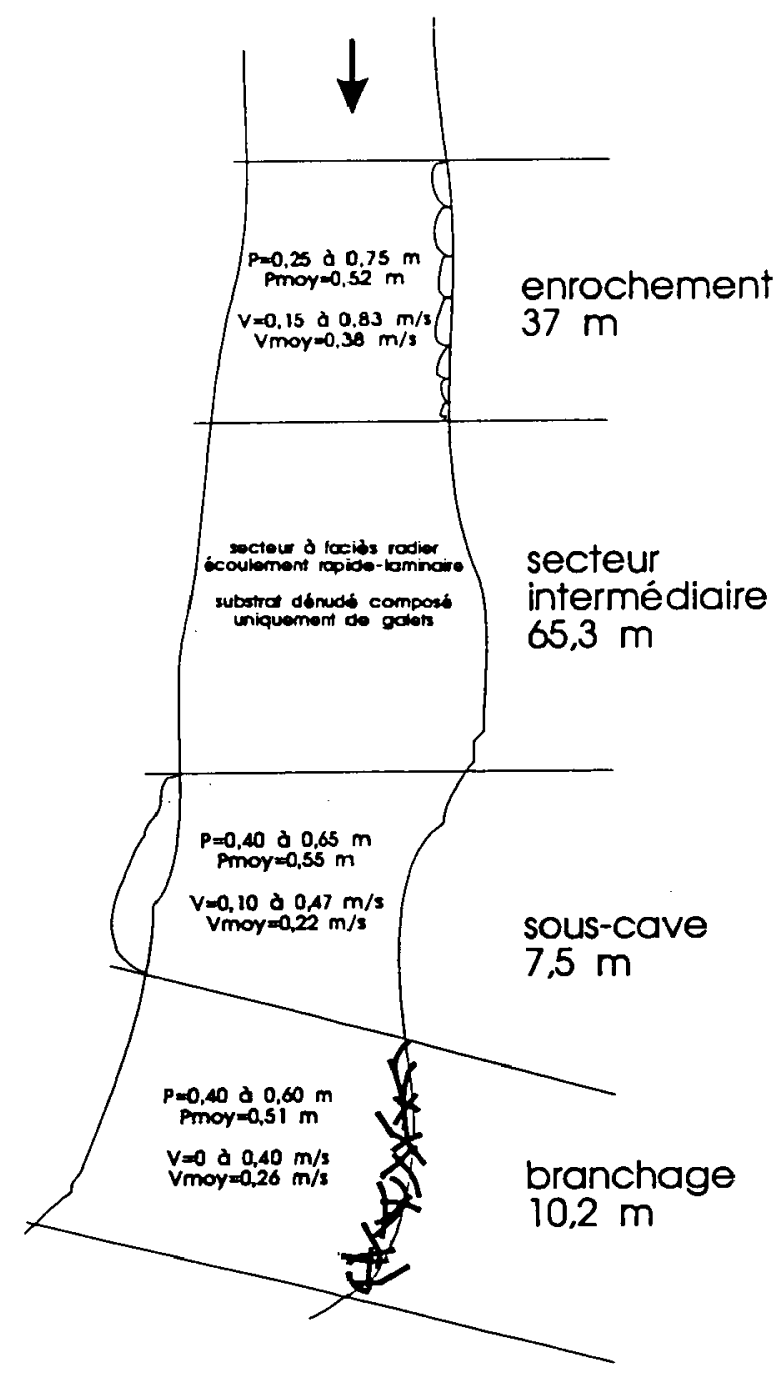

Figure 2 : Le GER - cartographie du secteur étudié.

Figure 2 : River GER - map of the study sector. 
Le déroulement de l'expérience peut se résumer comme suit :

a) Evaluation de l'état initial du secteur : la densité, la biomasse des poissons et la structure de la population sont déterminées par pêche électrique pour chaque type de cache et pour le reste du secteur correspondant à chacun de ces groupes de caches.

b) Suppression de l'accès aux trois groupes de caches : après avoir pêché et stocké les poissons dans les viviers, l'accès aux caches est supprimé le plus efficacement possible avec du grillage de vide de maille de $10 \mathrm{~mm}$. Une fois l'aménagement des caches effectué, les poissons sont libérés dans tout le secteur d'étude.

c) Réouverture progressive de l'accès aux différents types de caches : les grillages sont enlevés sur les caches BRANCHAGES, SOUS-CAVE et ENROCHEMENT. Après stabilisation du peuplement, on réalise une pêche électrique dans chaque cache et la portion de rivière correspondante : ceci permet d'estimer la recolonisation du secteur dont les caches ont été rouvertes.

L'estimation des superficies de chaque type de cache est donnée dans le tableau t.

\section{Tableau I : Superficie des secteurs étudiés.}

Table I : Surface area of the study sections.

\begin{tabular}{|c|c|c|c|}
\hline & SURFACE TOTALE $\left(\mathrm{m}^{2}\right)$ & SURFACE UTILE DES CACHES $\left(\mathrm{m}^{2}\right)$ & OBSERVATIONS \\
\hline EMROCHEMENT & $37 \times 6,5=240$ & $37 \times 2=74$ & $\begin{array}{l}\text { l'enrochement représente } 7,5 x \text { du } \\
\text { secteur et } 61 x \text { des caches }\end{array}$ \\
\hline SOUS-cave & $7,5 \times 7,2=54$ & $7,5 \times 1,5=11$ & $\begin{array}{l}\text { La zone sous-cave représente } 1,6 x \text { du } \\
\text { secteur et } 13 x \text { des caches }\end{array}$ \\
\hline BRANCHAGE & $11 \times 10,2=112$ & $10.2 \times 3=30$ & $\begin{array}{l}\text { La zane branchage représente } 3 x \text { du } \\
\text { secteur et } 25 x \text { des caches }\end{array}$ \\
\hline ENSEMBLE DU SECTEUR & $120 \times 8,2=984$ & $\begin{array}{l}74+11+30=115 \\
\text { + environ } 5 \mathrm{~m}^{2} \text { de cache bordure } \\
\text { (végétation) }=120\end{array}$ & $\begin{array}{l}\text { les caches représentent } 12,2 x \text { de la } \\
\text { surface totale du secteur }\end{array}$ \\
\hline
\end{tabular}

\section{CACHES ARTIFICIELLES (fig. 3)}

L'expérience s'est déroulée sur la rivière PIQUE en amont de Luchon (fig. 1). Le secteur choisi s'étend sur environ 240 mètres. II est délimité en amont et en aval par une chute de plusieurs mètres de hauteur, infranchissable pour les truites. Ce tronçon a été divisé en deux parties :

- La partie aval (zone d'étude), qui mesure 130 mètres, reçoit les caches artificielles ; elle a été choisie car elle recelait essentiellement des caches naturelles sous forme de petits blocs qui ont pu être enlevés sans moyens mécaniques considérables.

- La partie amont, longue d'une centaine de mètres, constitue la "zone tampon" et comporte de nombreuses caches naturelles inamovibles sous forme de gros blocs.

- Une zone intermédiaire de 20 mètres, sans cache, avec un substrat de graviers et de galets, barrée par un tronc d'arbre de $40 \mathrm{~cm}$ de hauteur, sépare les deux parties.

Le secteur d'étude est subdivisé en 13 sous-secteurs de 10 mètres de long. Cette subdivision a été effectuée pour faciliter la cartographie des caches pour les différentes séquences. Pour chaque sous-secteur, des mesures de vitesse de courant et de profondeur par transect de $10 \mathrm{~m}$ ont été réalisées (Tableau II).

Sept types de caches artificielles en poteries creuses (matériaux de construction) ont été retenus. Les caractéristiques de ces caches sont données dans le tableau III. Les séquences de disposition des caches s'organisent de la façon suivante :

- séquence "RIVES" : toutes les caches sont disposées près des rives,

- séquence "MILIEU" : toutes les caches sont disposées au milieu de la rivière,

- séquence "MIXTE" : les caches sont disposées indifféremment au milieu de la rivière ou près des rives, au hasard. 


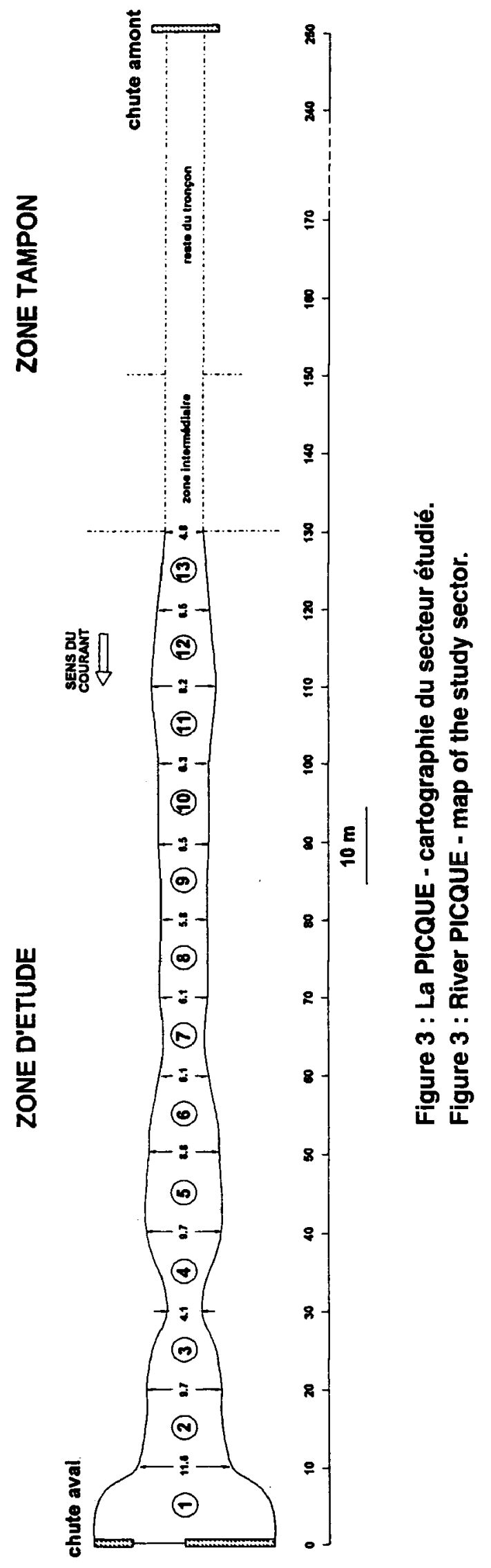


Tableau II : Profondeurs et vitesses dans les différents secteurs.

Table II : Water depth and velocity in study sections

\begin{tabular}{|c|c|c|}
\hline SECTEUR & VITESSE (DOSS) & PROFOAOELR (a) \\
\hline 1 & $0.15 \cdot 1.06$ & $0,20-2,50$ \\
\hline 2 & $0,30 \div 0,46$ & $0,15-0,25$ \\
\hline 3 & $0,13 \cdot 0,30$ & $0,10 \cdot 0,50$ \\
\hline 4 & $0,08 \cdot-0.33$ & $0,20-0,50$ \\
\hline 5 & $0.10 \cdot 0.30$ & $0,10 \cdot 0,15$ \\
\hline 6 & $0,10 \cdot 0,30$ & $0,10-0,20$ \\
\hline 7 & $0,05-0,35$ & $0,18-0,35$ \\
\hline 8 & $0,05 \cdot 0.52$ & $0,10-0,40$ \\
\hline 9 & $0,07 \cdot 0,70$ & $0,15-0,28$ \\
\hline to & $0,05-0,20$ & $0,10 \cdot 0,20$ \\
\hline 11 & $0,29-0,75$ & $0,10-0,22$ \\
\hline 12 & $0.46-0.56$ & $0,15-0,30$ \\
\hline 13 & $0,25 \cdot 0.56$ & $0.11-0,25$ \\
\hline
\end{tabular}

Tableau III : Surface utile des caches artificielles.

Table III : Useful surface area of artificial covers.

\begin{tabular}{|c|c|}
\hline TYPE DE CACHE & SURFACE UT ILE (m $)$ \\
\hline TUILE & 0,08 \\
\hline CLAUSTRE SAQCDIEN & 0,037 \\
\hline BRIOUE CREUSE & 0,08 \\
\hline BLOC DE COFFRAGE & 0,04 \\
\hline CLAUSTRE QUBJOUE & 0,06 \\
\hline COOUILLE & 0,063 \\
\hline APPUI FENETRE & 0,045 \\
\hline
\end{tabular}

Pour chaque séquence, les caches (composées de groupes de 2 à 7 éléments du même type) sont disposées dans chaque sous-secteur en essayant, autant que possible, de réaliser une disposition équivalente entre les sous-secteurs.

Exemple de la composition d'un sous-secteur : 2 groupes de 7 tuiles, 1 groupe de 2 claustres, 1 groupe de 2 coquilles, 1 groupe de 3 briques, 1 groupe de 2 briques de coffrage, 1 groupe de 2 appuis fenêtre, 1 groupe de 3 cubes.

Pour chaque séquence, trois journées de travail ont été nécessaires :

$1^{\text {er }}$ jour : vérification détaillée de la disposition des caches avec une remise en place si nécessaire suivant la cartographie détaillée.

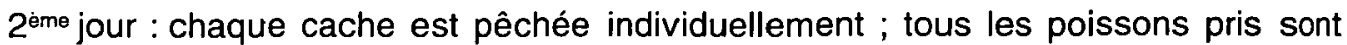
mesurés et notés cache par cache, puis reportés sur la carte. Après la pêche cache par cache, les poissons capturés étant mis de côté, une pêche systématique de tout le secteur d'étude est réalisée. Durant cette seconde pêche, tout le secteur étudié, y compris les caches, a été prospecté. Ceci permet de connaître le peuplement hors des caches.

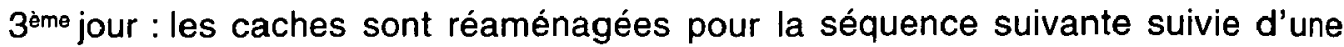
cartographie. 
Le matériel de pêche électrique utilisé est constitué par un groupe électrogène Electropullman avec cathode fixe et anode mobile :

- pour la pêche d'inventaire, nous avons utilisé la méthode de DE LURY : 2 passages successifs (420V, $2 \mathrm{~A})$,

- pour la pêche particulière (séquences RIVE, MILIEU, MIXTE), la prospection se fait d'abord au niveau de chaque cache (150 V, 1,5 à 2 A) puis du reste du secteur $(420 \mathrm{~V}, 2 \mathrm{~A})$.

\section{RÉSULTATS}

\section{CACHES NATURELLES}

Le secteur étudié possède une superficie de $948 \mathrm{~m}^{2}$ (120 m de long sur 8,20 m de large). Sur ce total, les caches représentent une surface de $120 \mathrm{~m}^{2}$, ce qui correspond à $12,2 \%$ de la surface totale. Les caractéristiques de chaque type de caches ainsi que les mesures morphodynamiques succinctes du secteur sont résumées dans la figure 2.

\section{- Etat initial (point 0) (tableau IV et fig. 4, 5, 8, 11)}

Les pêches d'inventaire du secteur étudié nous ont permis de constater que :

- la biomasse du secteur est de $278 \mathrm{~kg} / \mathrm{ha}$ correspondant à une densité de 2469 truites $/$ ha, soit $27,8 \mathrm{~g} / \mathrm{m}^{2}$ pour 0,2 truite $/ \mathrm{m}^{2}$. Cette valeur de biomasse peut être considérée comme forte par rapport aux cours d'eau de la région. Les populations du secteur étudié sont à la fois naturelles et artificielles comme dans tous ces cours d'eau ; naturelles, car il y a de nombreuses frayères en amont et en aval de ce secteur ; artificielles, par des déversements qui s'effectuent chaque année sous forme d'alevins de printemps $(5-6 \mathrm{~cm})$ et de truitelles d'automne $(10-15 \mathrm{~cm})$,

- par type de caches, la biomasse et la densité sont très importantes. Par ordre d'importance, on trouve : les SOUS-CAVE avec 7 truites $/ \mathrm{m}^{2}$ pour une biomasse de 732 $\mathrm{g} / \mathrm{m}^{2}$, les ENROCHEMENTS avec 1,3 truite $/ \mathrm{m}^{2}$ pour une biomasse de $179 \mathrm{~g} / \mathrm{m}^{2}$ et les BRANCHAGES avec 0,9 truite $/ \mathrm{m}^{2}$ pour une biomasse de $105 \mathrm{~g} / \mathrm{m}^{2} ; 83 \%$ des poissons ont été pêchés dans ces caches contre $17 \%$ hors des caches,

- la structure du peuplement est bien équilibrée avec deux pics importants : le premier entre 60 et $100 \mathrm{~mm}$ et le 2 ème entre 160 et $220 \mathrm{~mm}$. Enfin, $66 \%$ de la population est pêchable (taille légale de capture de $18 \mathrm{~cm}$ ).

\section{- Caches inaccessibles (caches cachées) (tab. V et fig. 6, 9, 12)}

Après avoir empêché les truites d'accéder aux caches en les barrant par des grillages, une pêche a été effectuée dans tout le secteur. Pour cette séquence, la biomasse et la densité sont deux fois moins importantes qu'elles ne le sont à l'état initial (125 kg/ha pour $1093 \mathrm{ind} / \mathrm{ha}$ ).

- Zone SOUS-CAVE/hors cache : la biomasse est seulement de $122 \mathrm{~g} / \mathrm{m}^{2}$ et la densité seulement de 1 truite $/ \mathrm{m}^{2}$ soit, par rapport au point 0 , une diminution de $31 \%$ en biomasse et $46 \%$ en densité.

- Zone ENROCHEMENT/hors cache : la biomasse est de $25,9 \mathrm{~g} / \mathrm{m}^{2}$ pour une densité de $0,19 \mathrm{ind} / \mathrm{m}^{2}$ soit, par rapport au point 0 , une diminution de $55 \%$ en biomasse et $63 \%$ en densité.

- Zone BRANCHAGES/hors cache : la biomasse est de $16,3 \mathrm{~g} / \mathrm{m}^{2}$ pour une densité de $0,24 \mathrm{ind} / \mathrm{m}^{2}$ soit, par rapport au point 0 , une diminution de $51 \%$ en biomasse et $22 \%$ en densité. 

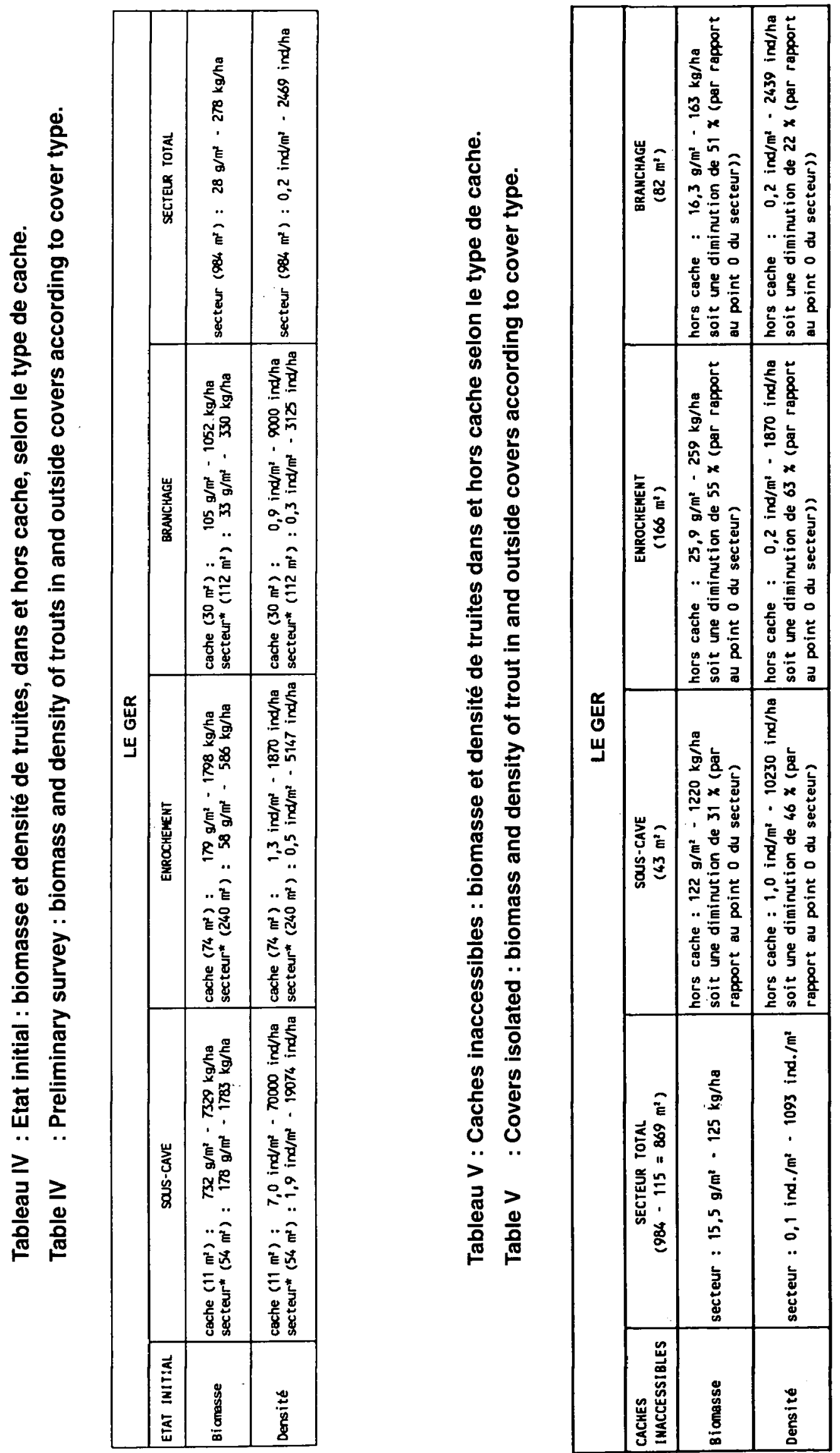


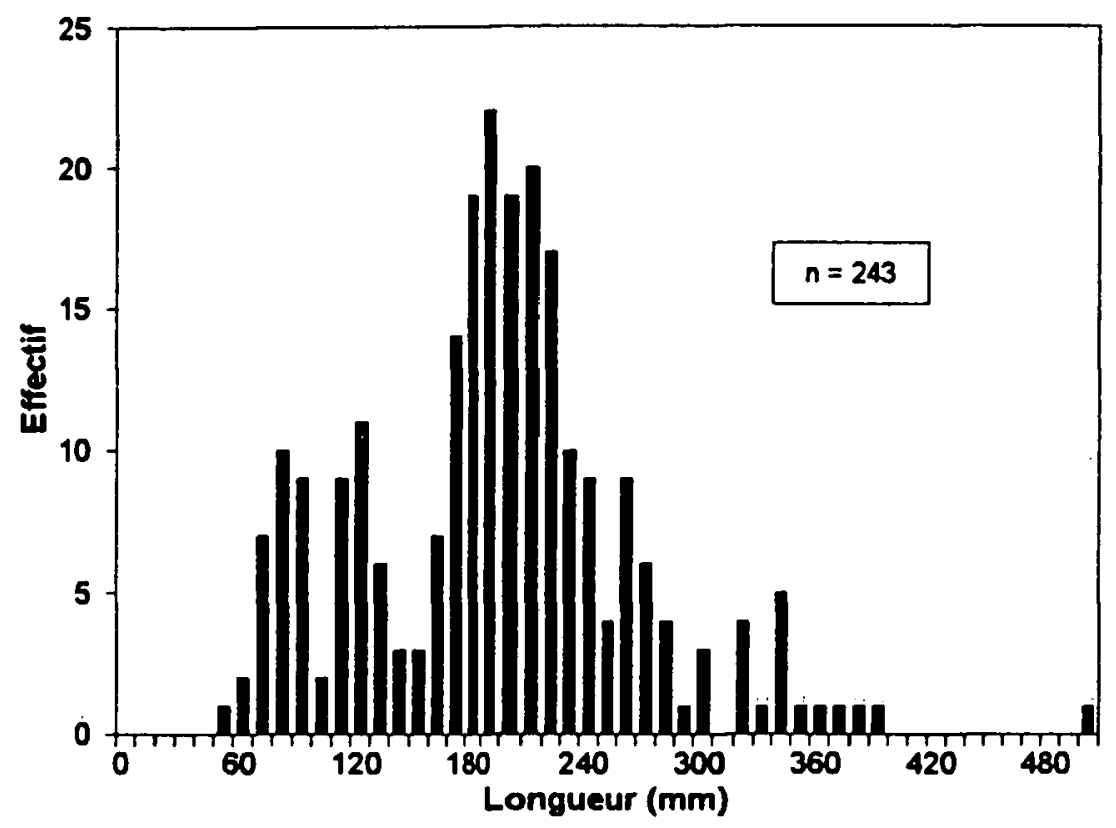

Figure 4 : Le GER - Histogramme des tailles des truites pêchées dans tout le secteur.

Figure 4 : River GER - Length-classes of trouts caught in the study sector.

POINT ZERO - SECTEUR SOUS/CAVE

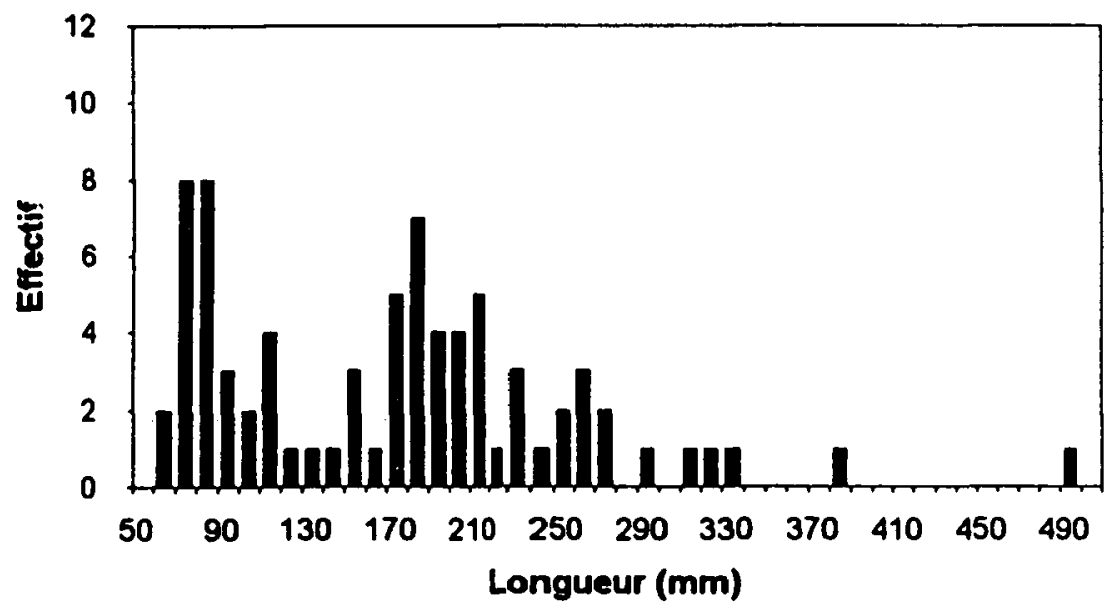

Figure 5 : Point zéro - Histogramme des tailles des truites pêchées dans le secteur sous-cave.

Figure 5 : Preliminary survey - Length-classes of trouts caught in the undercut bank section. 


\section{POINT ZERO - SECTEUR ENROCHEMENT}

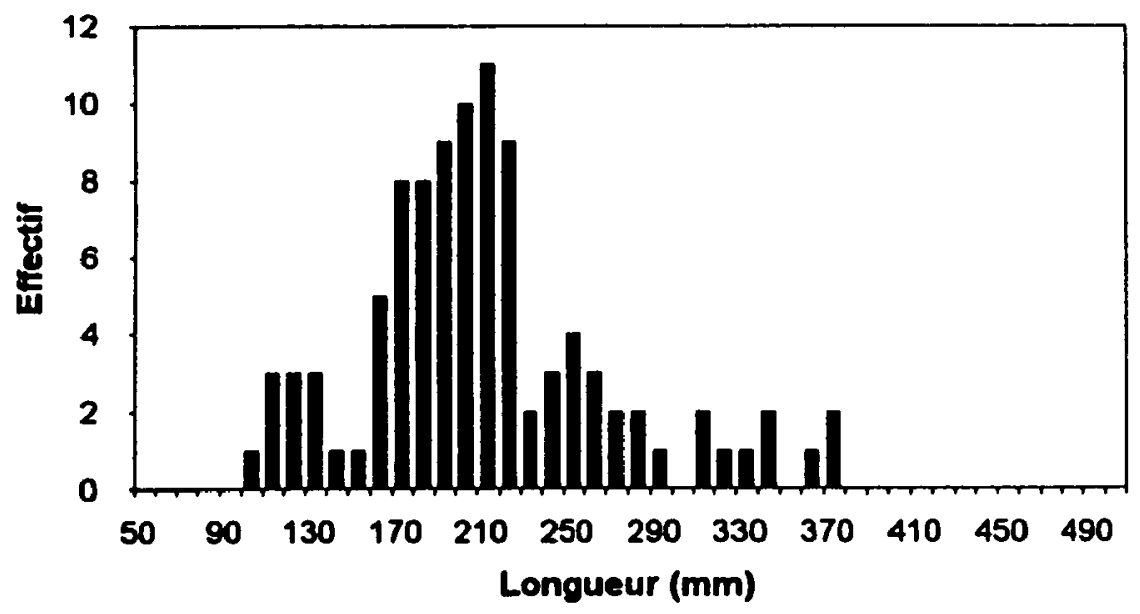

Figure 8 : Point zéro - Histogramme des tailles des truites pêchées dans le secteur enrochement.

Figure 8 : Preliminary survey - Length-classes of trouts caught in the rocky bank section.

\section{POINT ZERO - SECTEUR BRANCHAGE}

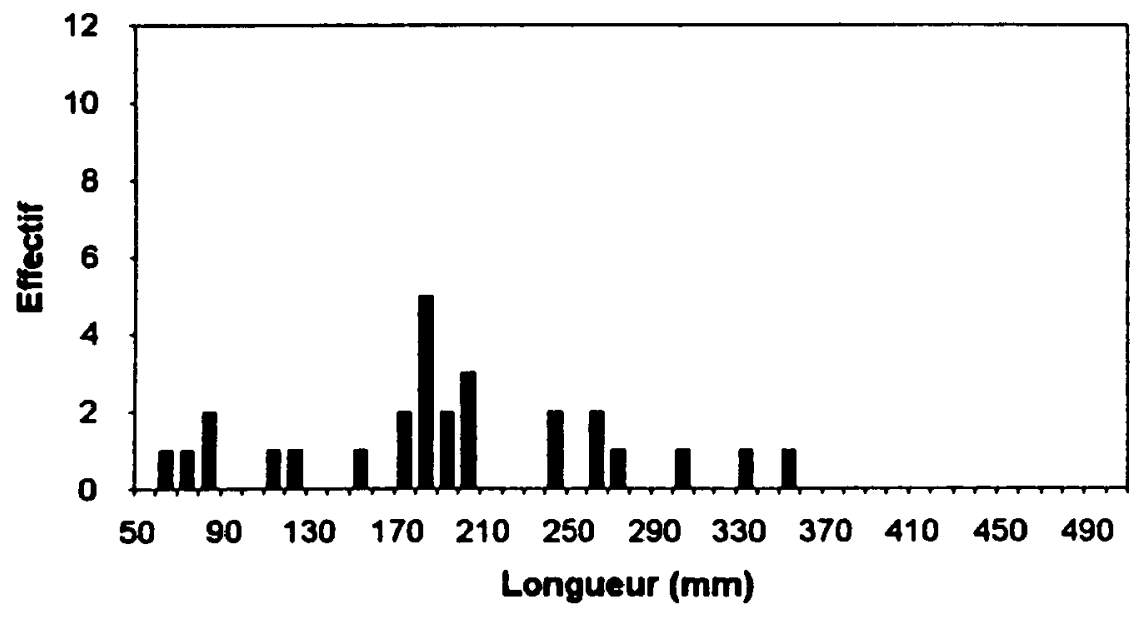

Figure 11 : Point zéro - Histogramme des tailles des truites pêchées dans le secteur branchage.

Figure 11 : Preliminary survey - Length-classes of trouts caught in the wood debris section. 
CACHES CACHEES - SECTEUR SOUS/CAVE

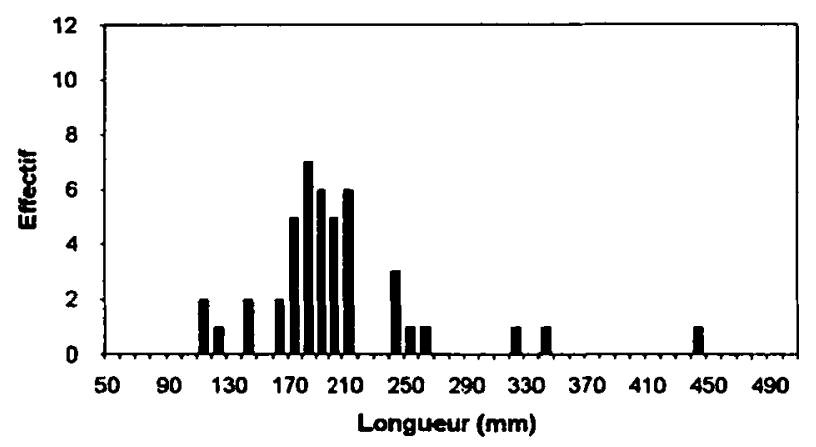

Figure 6 : Caches inaccessibles - Histogramme des tailles des truites pêchées dans le secteur sous-cave.

Figure 6 : Covers isolated - Length-classes of trouts caught in the undercut bank section.

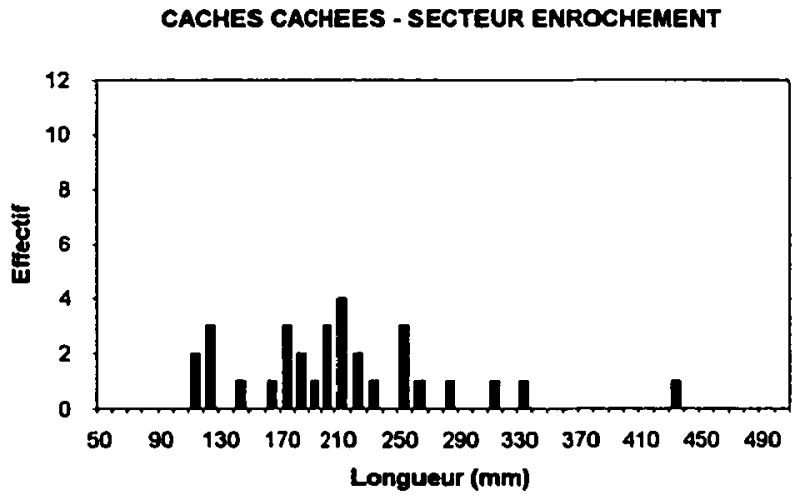

Figure 9 : Caches inaccessibles - Histogramme des tailles des truites pêchées dans le secteur enrochement.

Figure 9 : Covers isolated - Length-classes of trouts caught in the rocky bank section.

CACHES CACHEES - SECTEUR BRANCHAGE

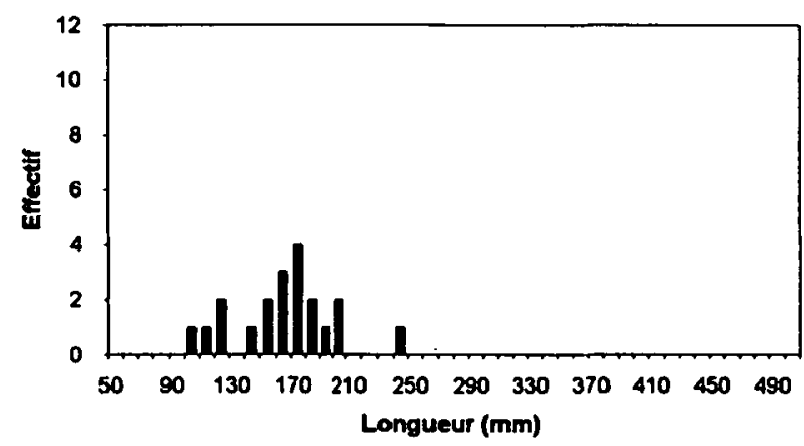

Figure 12 : Caches inaccessibles - Histogramme des tailles des truites pêchées dans le secteur branchage.

Figure 12 : Covers isolated - Length-classes of trouts caught in the wood debris section. 
- Recolonisation (tab. VI et fig. 7, 10, 13)

Vingt-et-un jours après la réouverture des caches, des pêches ont été effectuées pour quantifier la recolonisation des différentes caches.

- SOUS-CAVE : on constate que la biomasse est de $526 \mathrm{~g} / \mathrm{m}^{2}$ pour une densité de 3,09 truites $/ \mathrm{m}^{2}$; par rapport à l'état initial, ces valeurs ne constituent que $72 \%$ en biomasse et $44 \%$ en densité. Du point de vue de la structure du peuplement, l'examen de l'histogramme des classes de taille fait ressortir un nombre de truites de grande taille plus important que celui des individus de petite taille.

- ENROCHEMENT : on constate que la biomasse est de $68 \mathrm{~g} / \mathrm{m}^{2}$ de cache, ce qui représente $38 \%$ de la biomasse initiale, et que la densité, qui est de $0,58 \mathrm{ind} / \mathrm{m}^{2}$, ne représente que $45 \%$ de la densité initiale. Par rapport à la séquence intermédiaire (caches inaccessibles), la biomasse a plus que doublé (facteur de gain 2,6 ) et la densité plus que triplé (facteur de gain 3,2 ). La structure du peuplement reste similaire entre les trois expériences.

- BRANCHAGES : la biomasse dans la cache est de $44,8 \mathrm{~g} / \mathrm{m}^{2}$, ce qui constitue $43 \%$ de la biomasse initiale. La densité est de $0,56 \mathrm{ind} / \mathrm{m}^{2}$ soit $62 \%$ de la densité initiale. La phase de recolonisation pour cette cache concerne essentiellement les truites de petite taille et de taille moyenne.

Par rapport à la phase intermédiaire (caches inaccessibles), la recolonisation a un facteur de gain 2,8 en biomasse et 2,3 en densité.

Tableau VI : Recolonisation : biomasse et densité de truites dans et hors cache selon le type de cache.

Table VI : Recolonisation : biomass and density of trouts in and outside covers according to cover type.

\begin{tabular}{|c|c|c|c|c|c|}
\hline \multicolumn{6}{|c|}{ LE GER } \\
\hline \multirow[t]{2}{*}{ RECOLONISATION } & SOUS-CAVE & \multicolumn{2}{|c|}{ ENROCHEMENT } & \multicolumn{2}{|c|}{ BRANCHAGE } \\
\hline & $t+21 j$ & to & $t+21 j$ & to & $t+21 j$ \\
\hline \multirow{2}{*}{ Biomasse } & $732 \mathrm{~g} / \mathrm{m}^{2}$ & $179 \mathrm{~g} / \mathrm{m}^{2}$ & $68 \mathrm{~g} / \mathrm{m}^{2}$ & $105 \mathrm{~g} / \mathrm{m}^{2}$ & $45 \mathrm{~g} / \mathrm{m}^{2}$ \\
\hline & $\begin{array}{c}\text { recolonisation de la cache : } \\
72 x \text { de la biomasse par } \\
\text { rapport ì to }\end{array}$ & \multicolumn{2}{|c|}{$\begin{array}{l}\text { recolonisation de la cache : } \\
38 x \text { de la biomasse par } \\
\text { rapport a to }\end{array}$} & \multicolumn{2}{|c|}{$\begin{array}{c}\text { recolonisation de la cache : } \\
43 x \text { de la biomasse par } \\
\text { rapport to to }\end{array}$} \\
\hline \multirow{2}{*}{ Densité } & $1,3 \mathrm{ind} / \mathrm{m}^{2} \quad 0,6 \mathrm{ind} / \mathrm{m}^{2}$ & 7,0 ind $/ \mathrm{m}^{2}$ & $3,1 \mathrm{ind} / \mathrm{m}^{2}$ & $0,9 \mathrm{ind} / \mathrm{m}^{2}$ & $0,6 \mathrm{ind} / \mathrm{m}^{2}$ \\
\hline & $\begin{array}{l}\text { recolonisation de la cache : } \\
44 \% \text { de la densité par } \\
\text { rapport à to }\end{array}$ & \multicolumn{2}{|c|}{$\begin{array}{c}\text { recolonisation de la cache : } \\
45 x \text { de la densité par } \\
\text { rapport a to }\end{array}$} & \multicolumn{2}{|c|}{$\begin{array}{l}\text { recolonisation de lo cache: } \\
62 X \text { de la densité par } \\
\text { rapport a to }\end{array}$} \\
\hline
\end{tabular}




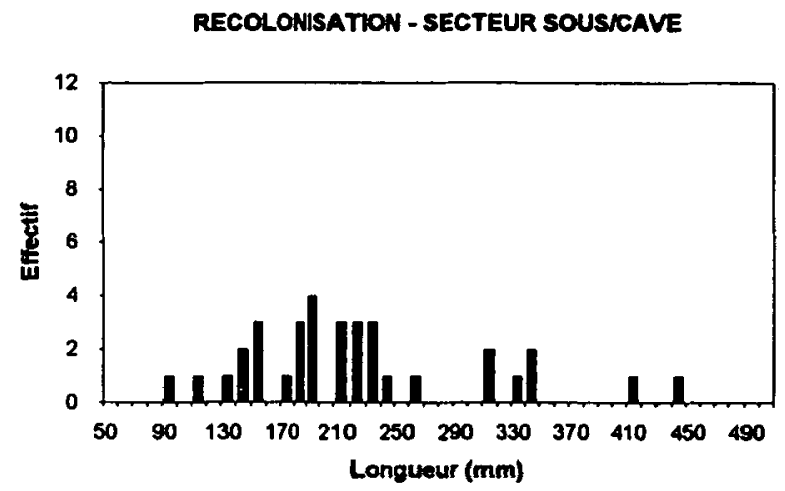

Figure 7 : Recolonisation - Histogramme des tailles des truites pêchées dans le secteur sous-cave.

Figure 7 : Recolonisation - Length-classes of trouts caught in the undercut bank section.

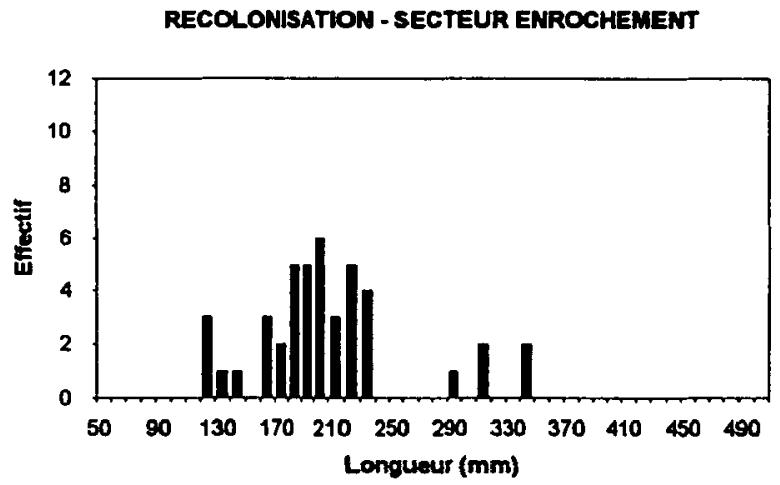

Figure 10 : Recolonisation - Histogramme des tailles des truites pêchées dans le secteur enrochement.

Figure 10 : Recolonisation - Length-classes of trouts caught in the rocky bank section.

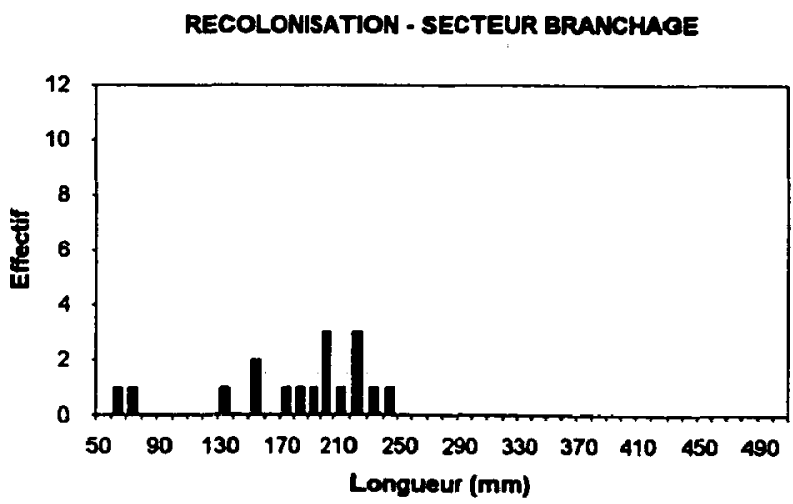

Figure 13 : Recolonisation - Histogramme des tailles des truites pêchées dans le secteur branchage.

Figure 13 : Recolonisation - Length-classes of trouts caught in the wood debris section. 


\section{Commentaires sur les caches naturelles}

L'étude réalisée sur les caches naturelles montre une hiérarchisation de la valeur des caches ; les préférenda des truites pourraient donc s'établir, en partant du meilleur type de cache, de la façon suivante:

a) SOUS-CAVE : la biomasse initiale est de $732 \mathrm{~g} / \mathrm{m}^{2}$ pour une densité de $7 \mathrm{ind} / \mathrm{m}^{2}$. S'il est privé de caches, ce secteur voit sa biomasse et sa densité chuter respectivement de $31 \%$ et de $46 \%$.

La recolonisation est la plus rapide si l'on compare aux autres types de caches. On notera que cette cache est recolonisée préférentiellement par de gros poissons. Après 21 jours de réouverture, la densité observée n'est que de $44 \%$ de sa valeur initiale contre $72 \%$ pour la biomasse.

b) ENROCHEMENT : la biomasse initiale est de $179 \mathrm{~g} / \mathrm{m}^{2}$ pour une densité de $1,3 \mathrm{ind} / \mathrm{m}^{2}$. En absence de cache, la biomasse et la densité du secteur chutent respectivement de $55 \%$ et $63 \%$ environ. La recolonisation (38\% pour la biomasse et $45 \%$ pour la densité, par rapport aux valeurs initiales) est plus faible que celle des caches sous-cave.

c) BRANCHAGES : parmi les trois types de caches étudiées, la colonisation de ce type de cache est la plus faible. Initialement, sa biomasse est de $105 \mathrm{~g} / \mathrm{m}^{2}$ correspondant à $0,9 \mathrm{ind} / \mathrm{m}^{2}$. En absence de cache, la biomasse et la densité du secteur chutent respectivement de $51 \%$ et $22 \%$ par rapport au point zéro.

L'ensemble du secteur étudié voit ainsi sa biomasse et sa densité chuter d'environ $55 \%$ lorsque l'accès aux 3 types de caches est fermé.

\section{CACHES ARTIFICIELLES}

\section{- Etat initial (point 0)}

Un inventaire piscicole, selon la méthode de DE LURY, a été effectué sur tout le tronçon étudié. Les résultats sont résumés ci-dessous :

Rivière : la PIQUE.

Secteur : amont Luchon - Date : 13 juillet 1990.

Longueur pêchée : $250 \mathrm{~m}$ - Largeur moyenne : 7,30 m - Surface pêchée : $1825 \mathrm{~m}^{2}$.

Densité/m² $: 0,27$ individu - Densité à l'hectare : 2767 individus.

Biomasse $/ \mathrm{m}^{2}: 5,9 \mathrm{~g}$ - Biomasse à l'hectare : $59,1 \mathrm{~kg}$.

Truites pêchables (taille légale de capture de $18 \mathrm{~cm}$ ) $: 9,4 \%$.

L'examen de l'histogramme montre que toutes les classes d'âge sont représentées, de l'alevin de l'année $(40 \mathrm{~mm}$ ) jusqu'aux individus de $280 \mathrm{~mm}$ (figure 14). La population de truites fario de ce secteur peut être considérée comme équilibrée. Cette population pourrait être considérée comme naturelle car il y a présence de nombreuses frayères dans le secteur étudié et, durant la période d'étude, aucun déversement n'a été effectué. 


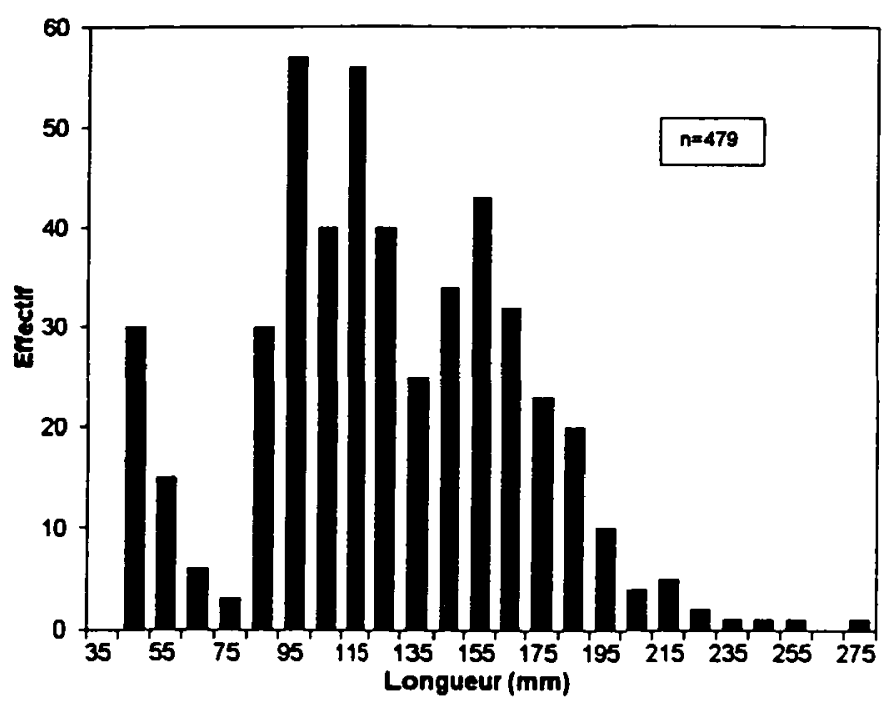

Figure 14 : La PIQUE - Histogramme des tailles des truites pêchées dans tout le secteur.

Figure 14 : River PIQUE - Length-classes of trouts caught in the study sector.

\section{- Séquence RIVES}

Pour cette séquence, les sept types de caches sont disposés sur les rives. Les résultats sont donnés dans les tableaux VII et VIII, et les figures 15 et 16 montrent la structure du peuplement des truites pêchées dans les caches et hors des caches.

Tableau VII : Séquence RIVE : surface totale des caches artificielles, nombre, poids, longueur moyenne, densité et biomasse des truites pêchées par type de cache.

Table VII : Covers near the bank : total surface area of artificial cover, number, weight, mean length, density and biomass of trouts caught per type of cover.

\begin{tabular}{|c|c|c|c|c|c|c|c|c|}
\hline SEOUENCE RNES & MOMBRE & SURF.UNHTNRE (m) & SURF.TOT (m) & NB. TRUITES & Pond (g) & TNLLE MOY. (mm) & DENSTIE (mAM) & BOMnSSE $(\mathrm{pmm})$ \\
\hline TULE & 100 & 0.08 & 15.1 & 103 & 2012 & 106 & 8.81 & 135.005 \\
\hline CLAUSTRE & 31 & 0.074 & 23 & 36 & 356 & $\boldsymbol{\infty}$ & 15.69 & 242.37 \\
\hline COQUMLE & 20 & 0.005 & 1.3 & 14 & 127 & 83 & 11.11 & 100.70 \\
\hline BAIOUE & 41 & 0.06 & 3.3 & 50 & $\infty 00$ & 104 & $\therefore \quad 17.07$ & 274,50 \\
\hline Q. COFFRAGE & 35 & 0.04 & 1.4 & 23 & 216 & os & $\begin{array}{r}16.4 \mathrm{~s} \\
\end{array}$ & 150.29 \\
\hline APPUIIF. & 17 & 0.000 & 1.1 & 19 & 409 & 113 & $\begin{array}{r}1734 \\
\end{array}$ & 637.91 \\
\hline CUBE & 4 & 0.08 & 29 & 20 & 304 & 96 & 6.00 & 120.81 \\
\hline TOtanx & 300 & & 28.4 & 27 & 4074 & 302 & & \\
\hline
\end{tabular}

Tableau VIII : Séquence RIVE : superficie globale des caches, nombre, pourcentage, densité et biomasse de truites dans et hors cache.

Table VIII : Covers near the bank : total surface area of covers, number, percentage, density and biomass of trouts in and outside cover.

\begin{tabular}{|c|c|c|c|c|c|c|}
\hline SEQUENCERNES & SUPERFICIE $(\mathrm{mm})$ & * SURFACE & NB TRUITES & * TRUITES & DENSITE (ind(m) & BIOMASSE (ghr") \\
\hline CACHES & 27.37 & 2.81 & 271 & 70.21 & 9.90 & 170.80 \\
\hline HORS CACHES & 945 & 97.19 & 115 & 29.79 & 0.12 & 2.19 \\
\hline SOUS-SECTEUR TOTAL & 972.37 & 100.00 & 386 & 100 & 0.40 & \\
\hline
\end{tabular}




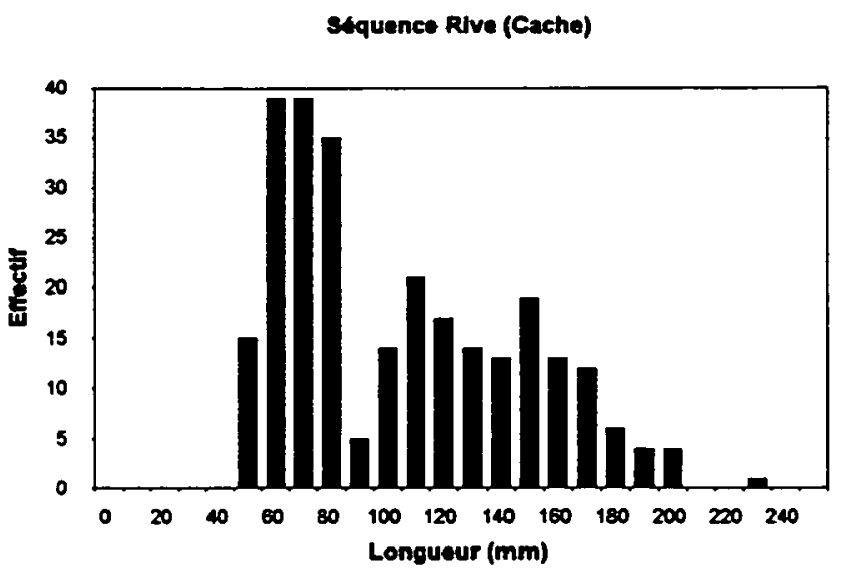

Figure 15 : Séquence RIVE - Histogramme des tailles des truites pêchées dans les caches.

Figure 15 : Covers near the bank - Length-classes of trouts caught in covers.

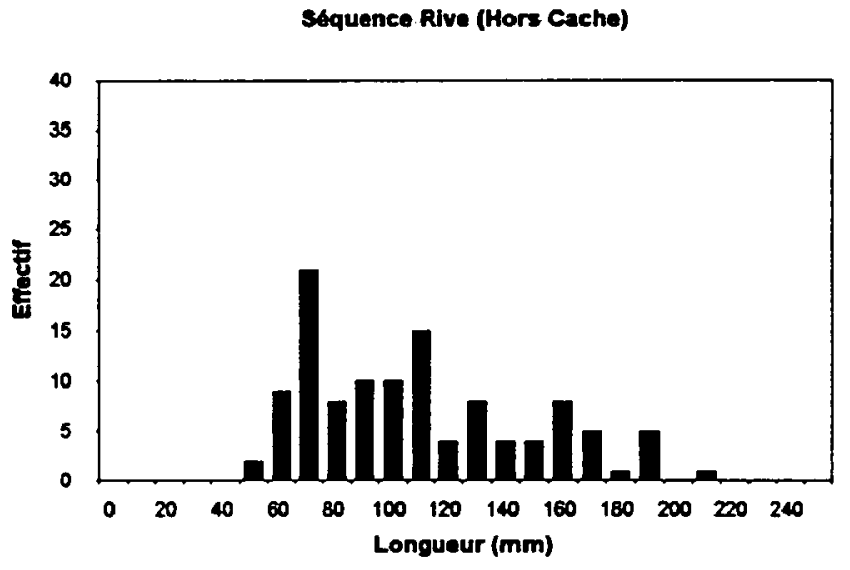

Figure 16 : Séquence RIVE - Histogramme des tailles des truites pêchées hors cache. Figure 16 : Covers near the bank - Length-classes of trouts caught outside cover.

La superficie totale des caches est de $27,5 \mathrm{~m}^{2}$, ce qui représente $2,8 \%$ de la surface mouillée du secteur étudié. Par type de cache, on compte $15 \mathrm{~m}^{2}$ pour les tuiles et une moyenne de $2 \mathrm{~m}^{2}$ pour chacun des six autres.

La densité, toutes caches confondues, est de :

$9,9 \mathrm{ind} / \mathrm{m}^{2}$ pour une biomasse de $170 \mathrm{~g} / \mathrm{m}^{2}$ de caches,

$0,12 \mathrm{ind} / \mathrm{m}^{2}$ pour une biomasse de $2,19 \mathrm{~g} / \mathrm{m}^{2}$ hors caches.

On compte, au total, $70 \%$ de poissons capturés dans les caches et $30 \%$ hors caches.

Par type de cache, on observe :

- BRIQUES, APPUIS FENETRE, BRIQUES de COFFRAGE et CLAUSTRES, avec une densité de truites comprise entre 15,7 et $17,7 \mathrm{ind} / \mathrm{m}^{2}$ pour une biomasse variant de 155 à $438 \mathrm{~g} / \mathrm{m}^{2}$.

- COQUILLES, avec une densité de $11 \mathrm{ind} / \mathrm{m}^{2}$ pour une biomasse de $100 \mathrm{~g} / \mathrm{m}^{2}$.

- CUBES et TUILES, avec une densité de $6,8 \mathrm{ind} / \mathrm{m}^{2}$ pour une biomasse de $129 \mathrm{~g} / \mathrm{m}^{2}$. 


\section{- Séquence MILIEU}

Les sept types de caches sont disposés uniquement au milieu de la rivière. Les résultats sont donnés dans les tableaux IX et $X$ et les figures 17 et 18 . La superficie totale des caches est de $19 \mathrm{~m}^{2}$, ce qui représente $2 \%$ de la surface mouillée du secteur étudié.

Tableau IX : Séquence MILIEU : surface totale des caches artificielles, nombre, poids, longueur moyenne, densité et biomasse des truites pêchées par type de cache.

Table IX : Covers in the middle of the bed : total surface area of artificial cover, number, weight, mean length, density and biomass of trouts caught per type of cover.

\begin{tabular}{|c|c|c|c|c|c|c|c|c|}
\hline SEOUENCE MLIEO: & NOMBRE & SURF.UNIT NRE (m) & SURF.TOT (m) & NQ. TRUITES & POADs (g) & TNLLE MOY. $(\mathrm{mm})$ & DENSTTE (mom) & BromussE (gtm) \\
\hline TULLE & 114 & 0.00 & 2.1 & 28 & 874 & 123 & 3.07 & 73.00 \\
\hline CLUSTRE & 20 & 0.074 & 21 & 13 & 336 & 128 & 6.06 & 150.57 \\
\hline COOUILE & 28 & 0.045 & 1.2 & 11 & 130 & 97 & 9.40 & 111.11 \\
\hline BRIOUE & 28 & 0.06 & 2.1 & 2 & 500 & 131 & 10.5s: & 278.65 \\
\hline B. COFFRACE & 37 & 0.04 & 1.5 & 17 & 456 & 128 & 11.49 & 300.11 \\
\hline APPUI F. & 16 & 0.060 & 1.0 & 11 & 170 & 110 & to.91 & 178.50 \\
\hline CUBE & 37 & 0.06 & 2.2 & 15 & 340 & 123 & 8.76 & 158.78 \\
\hline TOTAUX & 205 & & 19.2 & 117 & 2702 & 122 & & \\
\hline
\end{tabular}

Tableau X : Séquence MILIEU : superficie globale des caches, nombre, pourcentage, densité et biomasse de truites dans et hors cache.

Table X : Covers in the middle of the bed : total surface area of covers, number, percentage, density and biomass of trouts in and outside cover.

\begin{tabular}{|c|c|c|c|c|c|c|}
\hline SEQUENCE MILIEU & SUPERFICIE (m) & \% SURFACE & NB TRUITES & * TRUITES & DENSITE (indm) & BIOMASSE (g/m") \\
\hline CACHES & 19.22 & 1.99 & 117 & 58.50 & 6.09 & 140.55 \\
\hline HORS CACHES & 945 & 98.01 & 83 & 41.50 & 0.09 & 1.73 \\
\hline SOUS-SECTEUR TOTAL & 964.22 & 100.00 & 200 & 100 & 0.29 & 4.49 \\
\hline
\end{tabular}

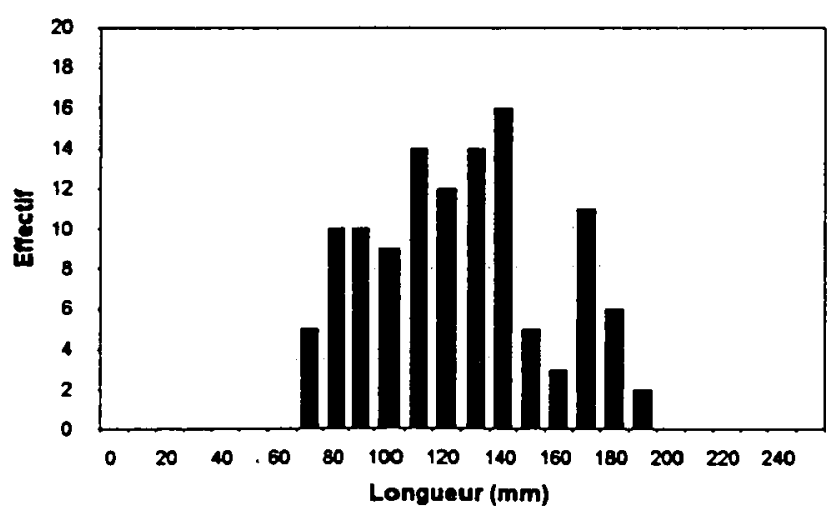

Figure 17 : Séquence MILIEU - Histogramme des tailles des truites pêchées dans les caches.

Figure 17 : Covers in the middle of the bed - Length-classes of trouts caught in covers. 


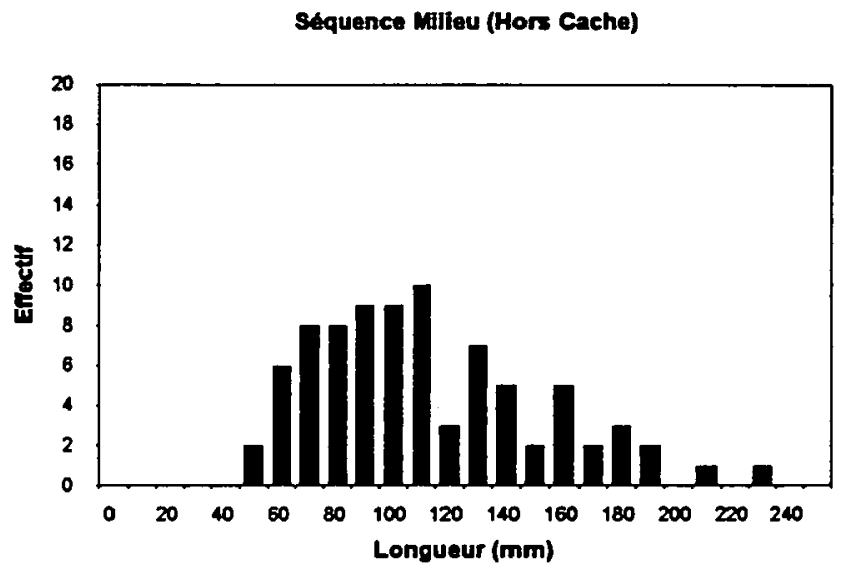

Figure 18 : Séquence MILIEU - Histogramme des tailles des truites pêchées hors cache.

Figure 18 : Covers in the middle of the bed - Length-classes of trouts caught outside cover.

La densité, toutes caches confondues, est de :

$6 \mathrm{ind} / \mathrm{m}^{2}$ pour une biomasse de $140 \mathrm{~g} / \mathrm{m}^{2}$ dans les caches, $0,09 \mathrm{ind} / \mathrm{m}^{2}$ pour une biomasse de $1,73 \mathrm{~g} / \mathrm{m}^{2}$ hors des caches.

On compte, au total, $58 \%$ de poissons capturés dans les caches et $42 \%$ hors des caches.

Par type de cache, on observe :

- BRIQUES, APPUIS FENETRE, BRIQUES de COFFRAGE, avec une densité comprise entre 10,5 et $11,5 \mathrm{ind} / \mathrm{m}^{2}$ pour une biomasse comprise entre 170 et $310 \mathrm{~g} / \mathrm{m}^{2}$.

- COQUILLES, CLAUSTRES et CUBES, avec une densité de 6 à 9 ind $/ \mathrm{m}^{2}$ pour une biomasse de 111 à $156 \mathrm{~g} / \mathrm{m}^{2}$.

— TUILES, avec une densité de 3 ind $/ \mathrm{m}^{2}$ pour une biomasse de $73 \mathrm{~g} / \mathrm{m}^{2}$.

\section{- Séquence MIXTE}

Les 7 types de caches sont disposés aussi bien sur les deux rives qu'au milieu du cours d'eau. Les résultats sont donnés dans les tableaux XI et XII et les figures 19 et 20.

La superficie utile des caches est de $28,5 \mathrm{~m}^{2}$, ce qui représente $2,9 \%$ de la surface mouillée du secteur.

La densité, toutes caches confondues, est de :

$12,5 \mathrm{ind} / \mathrm{m}^{2}$ pour une biomasse de $223 \mathrm{~g} / \mathrm{m}^{2}$ dans les caches,

$0,2 \mathrm{ind} / \mathrm{m}^{2}$ pour une biomasse de $2 \mathrm{~g} / \mathrm{m}^{2}$ hors des caches.

On compte, au total, $65 \%$ de truites capturées dans les caches, pour $35 \%$ hors des caches.

Par type de cache, on observe:

- BRIQUES et CLAUSTRES : densité supérieure à $20 \mathrm{ind} / \mathrm{m}^{2}$ pour une biomasse comprise entre 389 et $487 \mathrm{~g} / \mathrm{m}^{2}$.

- APPUIS FENETRE et BRIQUES de COFFRAGE : densité de $19 \mathrm{ind} / \mathrm{m}^{2}$ pour une biomasse comprise entre 300 et $322 \mathrm{~g} / \mathrm{m}^{2}$.

- COQUILLES et CUBES : densité 14 et 11 ind $/ \mathrm{m}^{2}$ pour une biomasse de $170 \mathrm{~g} / \mathrm{m}^{2}$.

- TUILES : densité $8 \mathrm{ind} / \mathrm{m}^{2}$ pour une biomasse de $142 \mathrm{~g} / \mathrm{m}^{2}$. 
Tableau XI : Séquence MIXTE : surface totale des caches artificielles, nombre, poids, longueur moyenne, densité et biomasse des truites pêchées par type de cache.

Table XI : Covers in the totality of the bed : total surface area of artificial cover, number, weight, mean length, density and biomass of trouts caught per type of cover.

\begin{tabular}{|c|c|c|c|c|c|c|c|c|}
\hline SEOUENCE WXXE & NOMBRE & SURF,UNITANRE (m') & SURF.TOT (m) & NB. JRUITES & Poros (g) & TALLLE MOY, (mm) & DENSITE (mohn) & Gomusse $(\mathrm{g} / \mathrm{m})$ \\
\hline TUILE & 198 & 0.08 & 15.6 & 125 & 2250 & 103 & 7.00 & 142.53 \\
\hline CLNUSTRE & 30 & 0.074 & 2.2 & 47 & 884 & 101 & 21.17 & 309.19 \\
\hline COOUILLE & 35 & 0.015 & 7.8 & 2 & 260 & 90 & 13.97 & 170.18 \\
\hline BRKOUE & 42 & 0.08 & 3.4 & 7 & 1650 & 110 & 20.51 & 407.50 \\
\hline 8. COFFRAGE & 37 & 0.04 & 1.5 & 27 & 44 & 101 & 10.24 & 300.00 \\
\hline APPUUI F. & 20 & 0.063 & 1.3 & 2 & 406 & $\$$ & 19.64 & 322.2 \\
\hline CUBE & 4 & 0.06 & 2.8 & 31 & 474 & 9 & 11.23 & 171.74 \\
\hline TOTAUX & 400 & & 20.5 & 386 & 6032 & 200 & & \\
\hline
\end{tabular}

Tableau XII : Séquence MIXTE : superficie globale des caches, nombre, pourcentage, densité et biomasse de truites dans et hors cache.

Table XII : Covers in the totality of the bed : total surface area of covers, number, percentage, density and biomass of trouts in and outside cover.

\begin{tabular}{|c|c|c|c|c|c|c|}
\hline SEQUENCE MUXTE & SUPERFICIE (mn') & * suRface & NB TRUITES & $\boldsymbol{*}$ TRUITES & DENSITE (indm) & B1OMASSE $(\mathrm{gm})$ \\
\hline CACHES & 28.50 & 2.93 & 356 & 64.61 & 12.49 & 222.92 \\
\hline HORS CACHES & 945 & 97.07 & 195 & 35.39 & 0.21 & 2.00 \\
\hline SOUS-SECTEUR TOTAL & 973.50 & 100.00 & 351 & 100 & 0.57 & 8.47 \\
\hline
\end{tabular}

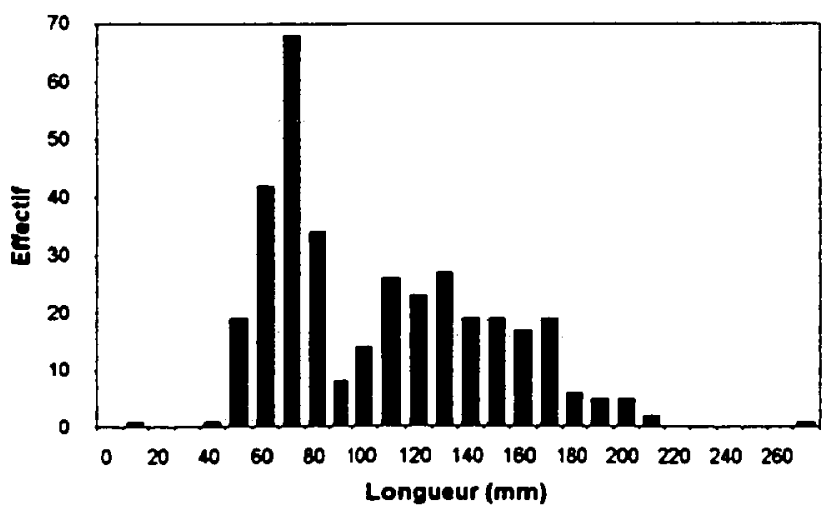

Figure 19 : Séquence MIXTE - Histogramme des tailles des truites pêchées dans les caches.

Figure 19 : Covers in the totality of the bed - Length-classes of trouts caught in covers. 


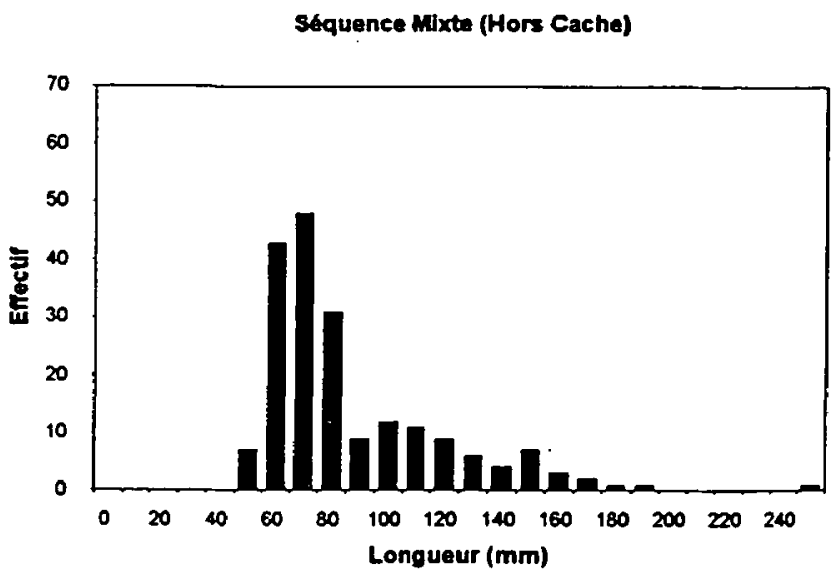

Figure 20 : Séquence MIXTE - Histogramme des tailles des truites pêchées hors cache.

Figure 20 : Covers in the totality of the bed - Length-classes of trouts caught outside cover.

\section{Commentaires sur les caches artificielles}

L'étude des caches artificielles, avec les séquences RIVE, MILIEU et MIXTE montre que :

- Suivant leur EMPLACEMENT, le taux de fréquentation des caches varie de 50 à $70 \%$. L'emplacement qui donne le meilleur résultat est la séquence RIVE $(70 \%)$ suivie de la séquence MIXTE (65\%), la moins bonne étant la séquence MILIEU (58\%). Dans tous les cas, 30 à $42 \%$ des truites fario se trouvent hors des caches.

- Suivant les types de caches, la densité varie de 3 à 23 truites par $\mathrm{m}^{2}$ de cache.

De toutes les séquences de pose confondues et par unité de surface $\left(\mathrm{m}^{2}\right)$, on peut classer les types de caches par ordre de préférence et pour une moyenne de 3 séquences:

- BRIQUES : $17 \mathrm{ind} / \mathrm{m}^{2}$ pour $346 \mathrm{~g} / \mathrm{m}^{2}$.

- APPUIS FENETRE : $16 \mathrm{ind} / \mathrm{m}^{2}$ pour $312 \mathrm{~g} / \mathrm{m}^{2}$.

- BRIQUES de COFFRAGE : $15 \mathrm{ind} / \mathrm{m}^{2}$ pour $254 \mathrm{~g} / \mathrm{m}^{2}$.

- CLAUSTRES : $14 \mathrm{ind} / \mathrm{m}^{2}$ pour $262 \mathrm{~g} / \mathrm{m}^{2}$.

- COQUILLES : $11 \mathrm{ind} / \mathrm{m}^{2}$ pour $126 \mathrm{~g} / \mathrm{m}^{2}$.

- CUBES : $6 \mathrm{ind} / \mathrm{m}^{2}$ pour $150 \mathrm{~g} / \mathrm{m}^{2}$.

— TUILES : $6 \mathrm{ind} / \mathrm{m}^{2}$ pour $117 \mathrm{~g} / \mathrm{m}^{2}$.

De ces résultats, une hiérarchie semble se dégager de ces différents types de caches artificielles, qu'on pourrait expliquer comme suit :

- BRIQUES, APPUIS FENETRE et CLAUSTRES semblent être les caches les plus appréciées des truites. Pour les BRIQUES, les résultats semblent logiques car ce type de cache, percé de trous longs mais petits en diamètre, brise bien le courant et est rapidement colonisé dans un premier temps par les macroinvertébrés et les truites par la suite. Pour les APPUIS FENETRE, la faible épaisseur de ces caches, qui se plaquent bien au fond dans la couche où la vitesse du courant est faible, peut expliquer la bonne colonisation obtenue.

- Pour les BRIQUES de COFFRAGE, la colonisation dépend de leur localisation (excellente dans la séquence MILIEU, et seulement en quatrième position dans la séquence MIXTE).

- Les caches les moins colonisées ont été en général les TUILES, les CUBES et les COQUILLES. Ces résultats sont assez prévisibles pour ces trois types de caches, car elles offrent peu d'abris face au courant et se fixent mal sur un substrat lisse. 


\section{CONCLUSION}

L'étude réalisée sur les caches nous permet de répondre aux objectifs fixés:

\section{CACHES NATURELLES}

Aux questions :

1. Quelle est la surface des caches naturelles sur une rivière pyrénéenne de faible altitude par rapport à la surface totale du secteur considéré ?

Réponse : Les caches (tous types confondus) représentent environ $12 \%$ de la surface mouillée d'un secteur considéré.

2. Quelles sont les densité et biomasse des truites dans les différents types de caches naturelles?

\section{Réponse :}

ENROCHEMENT (protection berge - abri courant) : $1,3 \mathrm{ind} / \mathrm{m}^{2}$ pour $179 \mathrm{~g} / \mathrm{m}^{2}$ SOUS-CAVE (creusement des berges avec racines d'arbres) : $7,0 \mathrm{ind} / \mathrm{m}^{2}$ pour $732 \mathrm{~g} / \mathrm{m}^{2}$. BRANCHAGES : $0, \hat{\vartheta}$ ind $/ \mathrm{m}^{2}$ pour $105 \mathrm{~g} / \mathrm{m}^{2}$.

Les préférences des truites vis-à-vis des caches sont les suivantes : SOUS-CAVE, ENROCHEMENT, BRANCHAGES.

3. Quelles sont les densité et biomasse des truites dans une rivière avec caches et sans cache?

Réponse : Secteur avec caches : 2469 ind/ha pour $278 \mathrm{~kg} / \mathrm{ha}$,

Secteur où les caches sont inaccessibles : 1093 ind/ha pour $125 \mathrm{~kg} / \mathrm{ha}$,

soit une diminution d'environ $55 \%$ en densité et en biomasse quand on enlève les caches dans un secteur de cours d'eau.

Nous rappelons que ces résultats proviennent de mesures immédiates, sans prédiction des effets à long terme qui pourraient être attendus.

4. Quelle est la recolonisation des truites après réouverture des caches ?

Réponse : Par rapport à la situation initiale, selon les types de caches, la recolonisation, après 21 jours de réouverture des caches, est estimée entre 38 et $72 \%$ pour la biomasse et entre 43 et $62 \%$ pour la densité.

La recolonisation tardive semble liée à la présence d'abris abondants en amont et en aval du secteur étudié. Ceci confirme le comportement sédentaire des truites constaté par MILLER (1957) qui enregistre $67 \%$ de recapture dans un même pool et estime la limite des territoires des truites à $200 \mathrm{~m}$, ceci étant valable pour les jeunes et pour les adultes (SOLOMON et TEMPLETON, 1976).

\section{CACHES ARTIFICIELLES}

Aux questions :

1. Quelle est la meilleure disposition des caches dans un cours d'eau où les caches naturelles sont absentes?

Réponse : Parmi les séquences testées, RIVE, MILIEU, MIXTE, les meilleurs résultats sont obtenus avec les séquences MIXTE et RIVE. Entre ces deux séquences, il y a très peu de différence et ceci est très important car, dans l'optique d'un aménagement de rivière par cache artificielle afin d'augmenter la densité et la biomasse, le gain de temps est appréciable si l'installation des caches se fait seulement près des rives. 
2. Quelles sont les préférences des truites pour les différents types de caches artificielles?

Réponse : Les BRIQUES et les APPUIS FENETRE semblent être les caches les plus appréciées des truites. Les densité et biomasse y sont plus élevées. Au point de vue pratique et économique, l'avantage est donné aux BRIQUES qui coûtent sept fois moins cher que les APPUIS FENETRE et présentent une surface utile et un poids assurant une bonne stabilité dans le cours d'eau.

3. Quelle est la densité des truites par mètre carré de cache?

Réponse : Suivant les types de caches et suivant les séquences, la densité des truites varie de 3 à $23 \mathrm{ind} / \mathrm{m}^{2}$. En dehors des caches, elle varie de 0,09 à $0,2 \mathrm{ind} / \mathrm{m}^{2}$. Il est donc indéniable que le rôle des caches est très important pour les truites, quel que soit leur stade de vie.

Malgré ces résultats très intéressants, nous émettons néanmoins quelques réserves concernant :

- l'efficacité de la pêche électrique, pour une telle étude, qui est toujours entachée d'un biais, si bien que l'on a tendance à surestimer la colonisation des caches ;

- la répétition de l'expérience, qu'on pourrait effectuer sur des cours d'eau moins productifs (biomasse et densité plus faibles) ;

- la répétition de l'expérience sur différentes époques de l'année ;

- un temps de stabilisation plus long, souhaitable entre les séquences d'étude.

En conclusion, l'installation de caches dans un secteur de cours d'eau est sûrement positive au niveau de la portion aménagée en apportant un gain de densité et de biomasse. Cependant, un bilan à long terme sur l'ensemble du cours d'eau pourrait montrer que, en fait, ces variations pourraient provenir de déplacements d'individus entre différents secteurs comme l'a constaté HUNT (1976).

Le choix électif des caches et leur rôle fondamental ne sont plus à démontrer mais les caches ne constituent qu'un maillon dans l'équilibre général de la rivière, les caches seules ne suffisent pas à corriger les effets perturbants provoqués par des aménagements physiques ou des altérations d'ordre hydraulique.

\section{REMERCIEMENTS}

Cette étude a été financée par EDF-DER à Chatou (Yvelines) dans le cadre de l'étude sur les débits réservés. Nous remercions la F.D.A.A.P.P. de la Haute-Garonne pour sa collaboration.

\section{BIBLIOGRAPHIE}

ALLEN K.R., 1969. Limitations on production in salmonid populations in streams. Symposium on Salmon and trout in stream. Northcote T.G. ed. H.R. McMillan Lectures in Fisheries, 3-18, Univ. of Brit. Col. Vancouver.

BACHMAN R.A., 1984. Foraging behavior of free-ranging wild and hatchery brown trout in a stream. Transactions of the American Fisheries Society, 113, 1-32.

BAIN M.B., FINN J.F., BOOKE H.E., 1985. Quantifying stream substrate for habitat analysis studies. N. Amer. J. Fish. Mgmt., 5, 499-506.

BELAUD A., CHAVEROCHE P., LIM P., SABATON C., 1989. Probability of use curves applied to brown trout (Salmo trutta L.) in river of Southem France. Reg. Riv., 3, 321-336.

BINNS N.A., EISERMAN F.M., 1979. Quantification of fluvial trout habitat in Wyoming. Trans. Amer. Fish. Soc., 108, 215-228.

BOUSSU M.F., 1954. Relationship between trout populations and cover on a small stream. Jour. Wildl. Mgt., 18, 229-239. 
BOVEE K., 1982. A guide to stream habitat analysis using the instream Flow Incremental Methodology. Inst. Flow Inf. Pap. 12, USDI Dish. Wildl. serv. FWS/OBS-82/26, 248 p.

BOWLBY J.N., ROFF F.C., 1986. Trout biomass and habitat relationships in Southern Ontario streams. Trans. Amer. Fish. Soc., 115, 503-514.

CUNJAK R.A., POWER G., 1986. Winter habitat utilization by stream resident brook trout (Salmo trutta). Can. J. Fish. Aquat. Sci., 43, 1970-1981.

DEVORE P.W., WHITE R.J., 1978. Daytime responses of brown trout (Salmo trutta) to cover stimuli in stream channel. Trans. Am. Fish. Soc., 107, 763-771.

GINGER R.D., 1973. Streamflow requirements of salmonids. Oregon Wildlife Commission, Job Final Repport, Project AFS 62-1, 117 p.

GRANT J.W.A., KRAMER D.L., 1990. Territory size as a predictor of the Upper Limit to population density of juvenile salmonids in streams. Can. J. Fish. Aquat. Sci., 47, 1724-1737.

GRANT J.W.A., NOAKES D.L.G., 1987. A simple model of optimal territory size for driftfeeding fish. Can. J. Zool., 65, 270-276.

HEGGENES J., 1988. Physical habitat selection by brown trout (Salmo trutta) in riverine systems. Nordic. J. Freshw. Res., 64, 74-90.

HEGGENES J., TRAAEN T., 1988. Daylight responses to overhead cover in stream channels for fry of four salmonid species. Holartic Ecology, 11, 94-201.

HUNT R.L., 1976. A long term evaluation of trout habitat development and its relation to improving management-related research. Trans. Am. Fish. Soc., 3, 361-364

McMAHON T.E., HARTMAN G.F., 1989. Influence of cover complexity and current velocity on winter habitat use by juvenile coho salmon (Oncorhynchus kisutch). Can. J. Fish. Aquat. Sci., 46, 1551-1557.

MILLER R.B., 1957. Permanence and size of home territory in stream-dwelling cutthroat trout. Journal of $t$ Research Board of Canada, 14, 687-691.

MILNER N.J., HEMSWORTH R.J., JONES B.E., 1985. Habitat evaluation as a fisheries management tool. J. Fish Biol., 27 (suppl. A), 85-108.

NÄSLUND I., 1989. Effects of habitat improvement on the brown trout, Salmo trutta L., population of a northern Swedish stream. Aquaculture and fisheries Management, $30,463-474$

NIELSEN G., 1986. Dispersion of brown trout (Salmo trutta L.) in relation to stream cover and water depth. Pol. Arch. Hydrobiol., 33, 475-488.

RALEIGH R.F., ZUCKERMAN L.D., NELSON P.C., 1986. Habitat suitability index models and instream flow suitability curves : brown trout. USDI Fish. Wildl. Serv. Biol. Rep., 82, 10-24.

SOLOMON D.J., TEMPLETON R.G., 1976. Movements of brown trout Salmo trutta L. in a chalk stream. J. Fish. Biology, 9, 411-423.

SOUCHON Y., TROCHERIE F., FRAGNOUD E., LACOMBE C., 1989. Les modèles numériques des microhabitats des poissons : application de nouveaux développements. Rev. Sci. Eau, 2, 777-792.

WESCHE T.A., GOERTLER C.M., HUBERT W.A., 1987. A modified habitat suitability index model for brown trout in Southeastern Wyoming. N. Amer. J. Fish. Mgmt., 7, 232-237.

WILZBACH M.A., 1985. Relative roles of food abundance and cover in determining the habitat distribution of stream-dwelling cutthroat trout (Salmo clarki). Can. J. Fish. Aquat. Sci., 42, 1668-1672. 